\title{
Visual Stylization and Emotional Analysis of Format Design
}

\author{
Bing Liu ${ }^{1, a}$, Ruiqiu Pang ${ }^{2, b,{ }^{*}}$ \\ ${ }^{1}$ Academy of Fine Arts, Northeast Normal University, Changchun130024, China \\ ${ }^{2}$ School of Geographical Sciences, Northeast Normal University, Changchun130024, China \\ aice8515@163.com, brq56789@yeah.net \\ ${ }^{\star}$ Corresponding author
}

Keywords: Format design, Visual stylization, Emotional.

\begin{abstract}
Format design is the overall art form of layout of various kinds of information in magazines, periodicals and advertisements. With the help of visual stylization and emotional shaping, excellent format design can effectively attract the audience's attention, cause the audience's emotional fluctuation, make the audience perceive the attitude and position of magazines, periodicals and advertisements to specific news events, and better perceive the personality and emotional color of the magazine, periodicals and advertisements. Format design can guide the audience to interpret the order of information, facilitate the audience to search for information of concern, and deepen the transmission of information by means of visual style and emotional expression.
\end{abstract}

\section{Introduction}

Format design is an important part of modern design art. Format design refers to the designer's purposeful arrangement and combination of visual elements, such as text and pictures, in accordance with the design intentions and visual needs, in a limited space, using the elements of shape and the principles of form [1, 2].

Format design is the overall artistic form of magazines, periodicals, advertisements, etc. Excellent format design can effectively attract the audience's attention. Through the layout, the audience can perceive the attitudes and feelings of magazines, periodicals and advertisements to the information transmitted, and can better perceive the characteristics and personality of the magazines, periodicals and advertisements. Format design mainly attracts the audience's vision, it uses people's visual and psychological perception to highlight the theme, and make information stand out, thus producing different visual effects [3].

Format design does not have a fixed category, but according to the needs of information transmission, free combination, forming its own unique style, it is convenient for readers to read and arouse the audience's visual experience, this is the unique charm of format design.

\section{Effective transmission of information and pursuit of visual impact}

When people appreciate painting works of art, they often pursue this rule, that is to say, first look at the whole picture, then observe the local details, which is the "center" of the picture. This theory is applied to format design, that is, to set the design center point, and further strengthen this center point, so that the audience can get peripheral information around this center, when they appreciate the works. In the market competition, magazines, periodicals and advertisements often use this "visual center", to make themselves the focus of many competing brands, and attract the attention of the audience [4].

\subsection{Recommendation and prominence of topic}

In the retail market, magazines, periodicals and advertisements are various, so it is necessary to put the most visually impacted pictures and titles on the cover in the format design, to play a strengthening role. Through the placement of key information on the cover, and the deepening and 
enlargement of headlines, "visual center point" has become the visual center of the audience. In the format design, we should strengthen the design of "visual center point", adopt a variety of format design, highlight the theme information, attract the attention of the audience, and achieve good publicity benefits.

\subsection{Selection and application of pictures}

Modern society is the era of "picture reading". The existence of pictures and words is the logo of modern excellent layout. With the development of the times, the role of pictures has become more and more obvious, in format design, the influence of pictures has become more and more significant. The choice of thematic pictures in magazines, periodicals and advertisements is very important, it can effectively convey thematic information, its color and connotation will not only attract the audience's attention, but also transmit the most information to the audience in the shortest time, thus stimulating the audience's purchasing power.

\subsection{Transmission and segmentation of information}

Magazines, periodicals and advertisements need to convey a lot of information. In format design, information can be segmented by certain design rules, for example, the lower part of the page highlights the information that the audience likes to receive, and promotes the transmission of information that people are not interested in on the upper part of the page; and with the help of interesting pictures, increase the title size and space occupied by the page, causing the audience to pay attention to the information.

In the format design, we can guide the flow of the audience's vision, with the help of unique lace forms, titles and pictures of the corresponding. In the design, the information is strengthened or segmented, by the arrangement of oblique lines or vertical lines, and by the comparison of light and shade, direction, size and straight lines. Through the layout of local and overall arrangements, better handling of the audience's line of sight guidance, so that the audience unconsciously in accordance with the requirements of the layout, to see what first, then what, and finally what to observe.

\section{The formation of the "vivid expression" of the page and the formation of visual beauty}

Excellent format design can make magazines, periodicals and advertisements have a "vivid expression", and leave a profound visual effect for the audience. It can make magazines, periodicals and advertisements full of rhythm sense, make the contents of the arrangement interesting, dynamic and fluent. To make the arrangement of titles and the selection of pictures match properly, to unify and change the contents and forms of magazines, periodicals and advertisements, to break the monotony and disorder of the layout, and to make the layout full of inspiration and beauty $[5,6]$.

\subsection{Strengthening of layout information}

Different magazines, periodicals and advertisements have different artistic charms and unique connotations. Modern format design is no longer a mere combination of lines and nets, it reflects the artistic characteristics of magazines, periodicals and advertisements, and conveys their attitudes towards news and information. Designers should enrich the art form of layout by means of art, strengthen the layout information, and enrich the unique charm of magazines, periodicals and advertisements: such as the atmosphere and solemnity of the news page, highlighting the weight and inherent shock of news information; the liveliness and joy of cultural and entertainment pages, with the help of pictures, show a strong cultural atmosphere and the tension and stimulation of entertainment; the passion of life layout, that is, people's relaxed and fashionable attitude towards modern life.

\subsection{Diversity of visual effects}

In modern format design, the change and unification of many elements is not only for the transmission of information, but also for the generation of visual aesthetics. In the process of format 
design, the unity of various visual elements can reflect the sense of order of format, promote the unity of the style of magazines, periodicals and advertisements, and form its own unique overall style. Format design deeply embodies the orientation and purpose of magazines, periodicals and advertisements, and then forms its own unique charm to attract the attention of the target masses. In format design, too many changes will only increase the burden of format design, make effective use of the "rhythm" of format design, increase visual aesthetics and promote the audience's understanding of information.

\section{Taking the flow of sight as the starting point, lighten the physiological and psychological pressure of vision}

Nowadays, information explosion society, in busy life, people will not receive too much information, therefore, the purpose of format design is to effectively transmit information in the shortest time, the shorter the process of information transmission, the better the effect of communication. Therefore, in the format design, we should adhere to the principle of simplicity and readability, reduce the visual and psychological pressure of the audience, reduce the visual fatigue of the audience, thus promoting the effective transmission of information. In format design, the elements of layout should be simplified, so that the audience can understand and accept information in the first time, and make the audience feel relaxed and happy.

In modern format design, modular arrangement can be adopted. In receiving information from magazines, periodicals and advertisements, audiences are accustomed to reading simply, that is, in the sight, they consciously organize and browse the meaningful and similar text and picture information, and interpret the information in an irregular way. In the format, we should consciously guide the flow of the audience's vision, that is, to place the manuscripts with similar or opposite meanings in the format consciously, and guide the audience to read the order of information. To make the format design concise, clear and neat, which is conducive to the audience to search for information of concern, easy to read and understand smoothly.

\section{Analysis of the development trend of modern layout design}

\subsection{Emphasis on creativity}

The innovation of the main body and the ingenious combination of editing techniques, are the developing trend of modern layout design. In magazines, periodicals and advertisements, format design has strong expressive force, which can give vivid and intuitive artistic expressive force to words and pictures. To achieve the best combination of words and pictures, and then effectively convey information, promote the interaction between design and audiences' ideas, inject vitality and interest into design, and achieve the perfect combination of form and content.

\subsection{Manifestation of individuality}

The display of individuality is the fashion trend of the art world today. The flexible use of format design can endow magazines, periodicals and advertisements with novel and unique personality performance, and absorb the attention and resonance of the audience. In the format design, the ingenious combination of pictures and words can make magazines, periodicals and advertisements get rid of the old and mediocre feeling, inject new vitality into the design, and have magical and ingenious visual effects.

\subsection{Capture of emotional}

Capturing the audience's emotions is a higher level artistic expression of format design. Modern design advocates humanized design, that is, respecting the feelings of the audience, "moving with emotion" is not only reflected in the information itself, but also in the atmosphere created by the arrangement and design of words and pictures. The combination of dots, lines and surfaces can promote the emotional factors of the audience's psychology, create the artistic aesthetic feeling of 
"lightness, dignity, ease and excitement", and embody the enthusiasm, rambling and free artistic style of magazines, periodicals and advertisements.

\section{Conclusion}

In magazines, periodicals and advertisements, format design has a strong expressive force, its basic function is not only the transmission of information, but also the comprehensive expression of visual style and emotion. This comprehensive expression can continuously optimize the structure and mode of information dissemination, and give magazines, periodicals and advertisements unique style charm and artistic appeal. Flexible use of format design can reflect the enthusiasm, rambling and free artistic style of magazines, periodicals and advertisements, and can guide the audience to interpret the order of information. Format design is conducive to the audience to search for information of concern, read conveniently, understand smoothly, and cause the audience's emotional fluctuations, and then truly establish and attract their own audience for a long time.

\section{Acknowledgements}

The author gratefully thanks the financial support by Fundamental Research Funds for the Central Universities / Youth fund of Northeast Normal University in philosophy and social science projects (2017QT008).

\section{References}

[1] Bing Liu, Hongtao Zhou, Analysis on the Laws of Formal Beauty in Format Design, Social Science and Humanity, Advances in Education Research, vol. 121, pp. 191- 194, 2018.

[2] Molly Bang, Picture This 25th Anniversary Edition: How Pictures Work, Chronicle Books; Anniversary, Expanded, Revised, 2016

[3] Sasaki Takashi, Layout design principle, China Youth Press, 2007.

[4] Ma Jianhua, Visual Flow in Format Design, Packaging engineering, vol. 6, pp. 191-193, 2018. (In Chinese).

[5] Yan chuan, Application of "orderliness" in layout design, Art Panorama, vol. 3, pp. 131, 2016. (In Chinese).

[6] He Baiyun, Discussion on book layout design, Journal of Nanjing Arts Institute (Arts and Design), vol.1, pp. 161-163, 2014. (In Chinese). 